\title{
Carotid Endarterectomy General or Regional Anesthesia
}

E.F.Makram, M.F.El Meliegy and M.Z.Amer

Anesthesia and Intensive Care Dept., Faculty of Medicine, Benha Univ., Benha, Egypt

E-Mail: mohamedzaki@gmail.com

\begin{abstract}
Carotid artery disease is the result of atherosclerosis, if the obstruction of common carotid and the bifurcation of the common carotid artery or internal carotid artery occurred. The embolic phenomenon is the main cause of this obstruction. The role of collateral blood flow (circle of Willis and exracranial anastomosis channels) is very important. During endoarterctomy, the degree of ischemia and postoperative complications depends on the degree of the dependency of cerebral circulation on the ipsi lateral internal carotid artery, and the efficiency of the collateral circulation. There is five folds increase in the incidence of perioperative stroke, with the presence of good cerebral blood flow during the surgery. The aim of this study is to review the anesthetic choices between general anesthesia and regional anesthesia displaying the merits of local anesthetic methods in achieving a reduced hospital stay, patient satisfaction, and fewer complications. Carotid artery disease is defined by the narrowing or lockage of the artery due to laque build-up. The process hat blocks these arteries (atherosclerosis) are basically the same as that which causes coronary artery disease and peripheral artery disease (PAD). The slow build-up of plaque (which is a deposit of cholesterol, calcium, and other cells in the artery wall) is caused by high blood pressure, diabetes, tobacco use, high blood cholesterol and other modifiable risk factors. Carotid endarterectomy is surgery to remove fatty deposits (plaque) that are narrowing the arteries in neck. These are called the carotid arteries.
\end{abstract}

Keywords: Carotid Artery Disease, Atherosclerosis, Endoarterctomy, Ischemia, Plaque, Collateral Circulation.

\section{Introduction}

Carotid artery disease is the result of atherosclerosis, if the obstruction of common carotid and the bifurcation of the common carotid artery or internal carotid artery occurred. The embolic phenomenon is the main cause of this obstruction. The role of collateral blood flow [circle of Willis and exracranial anastomosis channels] is very important.

During endoarterctomy, the degree of ischemia and postoperative complications depends on the degree of the dependency of cerebral circulation on the ipsi lateral internal carotid artery, and the efficiency of the collateral circulation. There is five folds increase in the incidence of perioperative stroke, with the presence of good cerebral blood flow during the surgery [1].

Ultrasonography-guided local anesthesia for CEA may be the gold standard, and when local anesthesia is used, re-operation can be performed without additional anesthetic [2] .

for patients who received cervical block anesthesia rather than general anesthesiawas reported previously, the difference did not reach statistical significance. In another paper dealing with carotid endarterectomy under generalanesthesia, only $50 \%$ of patients had one day postoperative stay, the othersleft the hospital later. Since early discharge is multifactorial, we studied riskfactors for staying longer than one day, including type of anesthesia [3].

However, benefit requires a low operative risk, which may depend on the type of anesthetic used. In our previous Cochrane review of several small, randomized control trials [RCT] of carotid endarterectomyusing local anesthesia [LA] vs general anesthesia published during 1966 to 2007, there was a trend toward lower operative mortality with LA and no difference in risk of strokebut statistical power was limited [4].

\section{Anatomy of carotid artery}

The common carotid artery is a paired structure, meaning that there are two in the body, one for each half. The left and right common carotid arteries follow the same course with the exception of their origin. The right common carotid originates in the neck from the brachiocephalic trunk. The left arises from the aortic arch in the thoracic region and can be thought of as having two parts: a thoracic [chest] part and a cervical [neck] part. But the right originates in or close to the neck, so contains a small thoracic portion [5].

\subsection{Carotid sinus}

A localized dilatation is present at the bifurcation of common carotid artery or the beginning of internal carotid artery, known as the carotid sinus. The tunica adventitia is thick while the tunica media is thinner, as compared to other parts. A number of nerve endings of the glossopharyngeal nerve are also found in the tunica adventitia. Thus carotid sinus serves as a receptor, detecting changes in the blood pressure. A rise causes vasodilatation and reduces the heart rate [5].

\subsection{The carotid body}

It is located within the neck, and in close approximation to the carotid bifurcation. It is composed of a number of chemoreceptor cells and supporting matrix and detects changes in the composition of blood in the common carotid as it forms the internal and external carotid arteries. It predomintly detects the partial pressue of oxygen, but is also sensitive to the partial pressure of $\mathrm{CO} 2$, $\mathrm{pH}$ and temperature [6]. 


\section{Carotid artery diseases}

Carotid artery disease is defined by the narrowing or lockage of the artery due to laque build-up. The process hat blocks this artery [atherosclerosis] is basically the same as that which causes coronary artery disease and peripheral artery disease [PAD]. The slow build-up of plaque [which is a deposit of cholesterol, calcium, and other cells in the artery wall] is caused by high blood pressure, diabetes, tobacco use, high blood cholesterol and other modifiable risk factors [7].

Over time, this narrowing may eventually become so severe that a blockage decreases blood flow to the brain and may tragically ause a stroke. A stroke can also occur if a piece of plaque or a blood clot breaks off from the wall of the carotid artery and travels to the smaller arteries of the brain [7].

\subsection{Risk factors}

Carotid artery disease is part of the arterial circulatory system and has similar risk factors as PAD and coronary heart disease: Family history of atherosclerosis [build-up of plaque in the peripheral, coronary or carotid arteries], Age [men have a higher risk before age 75 , women have a higher risk after age 75], Smoking, Hypertension, Diabetes and High cholesterol, and especially high amounts of "low density lipoprotein" [or LDL, the bad form of cholesterol]; although this risk factor appears to be less strong for stroke than it is for coronary artery disease [7].

\subsection{Symptoms}

As for all artery diseases, there are usually no advanced warning signs for early forms of carotid artery disease. For many individuals, the first obvious sign often is a TIA or mini-stroke. Symptoms for a stroke or TIA are similar and may include blurring, dimming, or loss of vision; tingling around the mouth, difficulty with speech, the inability to normally move an arm or leg, the inability to feel [numbness] in a part of the body and rarely, a sudden severe headache. [7].

\subsection{Diagnosis}

Proteins in the wall of the aorta, called elastin and collagen the diagnosis of carotid artery disease is usually based on an ultrasound examination of the neck arteries [a carotid artery duplex scan]. Alternatively, the artery can be visualized by a magnetic resonance angiogram [MRA] or standard angiogram [7].

\subsection{Treatment}

Treatment for carotid artery disease normally consists of normalization of those risk factors that cause artery blockages, specific medications [usually antiplatelet medications], and sometimes treatment to open the narrowed carotid artery with an angioplasty and stent, or by a surgical procedure.

Health care providers will want to reduce risk for developing blood clots in order to prevent stroke or heart attack. A daily antiplatelet medication, such as aspirin, Plavix [clopidogrel], Aggrenox [aspirin combined with dipyridamole], or warfarin may be prescribed. The choice of medication is one that is best made by your own health care provider. Individuals with severe blockages of the carotid artery [usually at least 6070 percent blockage] may be recommended for a surgical treatment called carotid endarterectomy [7].

A new "nonsurgical" endovascular treatment uses angioplasty and stents to open blocked carotid arteries. This procedure's safety and efficacy continues to be studied in several medical centers. This procedure involves the placement of a small flexible tube [catheter] into an artery from the groin. The catheter is then directed to the neck to reach the carotid artery blockage. A balloon pushes open the artery wall and a stent [a small metallic coil] is often left to keep the artery open [7].

\subsubsection{Medical treatment}

Lowering blood pressure to a target below $120 / 80 \mathrm{~mm} \mathrm{Hg}$ by life style interventions and antihypertensive treatment is recommended in persons who have had an ischemic stroke or transient ischemic attack [TIA] and are beyond the hyperacute period. Angiotensin-converting enzymes and angiotensin receptor blockers are recommended as first-choice medications for patients with diabetes. Glucose control to nearnormoglycemic levels [target hemoglobin A1C $7 \%$ ] is recommended among diabetics to reduce microvascular complications and, with lesser certainty, macrovascular complications. Counseling and smoking cessation medications have been found to be effective in helping smokers to quit. Lower quality evidence suggested possible benefits of avoiding environmental tobacco smoke, reduction of alcohol consumption by heavy drinkers, weight reduction for obese patients, and increasing physical activity. Antiplatelet agents are recommended for patients with noncardioembolic ischemic stroke or TIA. Aspirin [50 to $325 \mathrm{mg} / \mathrm{d}$ ], the combination of aspirin and extended-release dipyridamole, and clopidogrel are all acceptable options for initial therapy [8].

\subsubsection{Carotid endareterectomy}

The endarterectomy is begun by carefully developing a subadvential plane with a freer dissector in the common carotid artery, completed circumferentially, feathered to a good end-point proximally and continued distally, everting the plaque out of the external carotid artery and then completed in the internal carotid artery where the plaque transitions into normal intima. Today, most 
evidence strongly supports arteriotomy closure with an autogenous vein, Dacron, or polytetrafluoroethylene patch using a running 6-0 polypropylene suture [9].

Alternatively, eversion endarterectomy is performed by obliquely amputating the internal carotid artery at the common carotid bifurcation and rolling back the adventitial layer until normal intima is recognized distally at the distal endpoint. Residual plaque in the common and external carotid arteries is endarterectomized at this time. After completion of the endarterectomy, the internal carotid artery is re-anastomosed to the common carotid artery with a running 6-0 polypropylene suture [9].

\subsubsection{Carotid artery stenting}

Carotid artery stenting is performed under local anesthesia with mild or no sedation. Patients are placed on clopidogrel and aspirin. Arterial access is achieved through a retrograde femoral artery approach. Brachial, radial, or direct CCA approaches have been used in some instances. Noninvasive or angiographic arch assessment assists in guiding the optimal approach to the CCA. Patients are heparinized to an activated clotting time [ACT] of 250300 seconds.

Post-stenting angioplasty is performed with a balloon undersized by $20 \%$ to $40 \%$ of CA diameter and the stent length. A moderate residual stenosis [20\% to $30 \%]$ is generally acceptable since continued expansion of nitinol stents may show additional luminal recruitment over time. Finally, the EPD is removed over a retrieval catheter. The completion angiogram must visualize the extra-and intracranial circulation in two or more views. The sheath is removed when the ACT is 150 seconds; arterial closure devices can be used to obviate the need for normalization of the ACT. Patients are placed on clopidogrel for at least 4 weeks and on aspirin indefinitely [9].

\section{Carotid endarterectomy}

Carotid endarterectomy is surgery to remove fatty deposits [plaque] that are narrowing the arteries in neck. These are called the carotid arteries. They supply blood and oxygen to brain. If plaque and other fatty materials block an artery, it slows or blocks the blood flow [10].

The asymptomatic carotid surgery trial [ACST] recruited patients with a carotid artery diameter reduction of at least $60 \%$ on ultrasound and no symptoms in the previous 6 months. This study showed a net reduction of $5.4 \%$ in the combined outcome of stroke within $5 \mathrm{yr}$ and death within 30 days of surgery [11].

The benefits were mainly seen in younger patients and were uncertain for patients older than 75 yr. The American Academy of Neurology documented that there is a significant difference between the primary endpoint of the ACAS and
ACST studies. ACAS took ipsilateral stroke as the primary endpoint, whereas ACST included contralateral and vertebrobasilar strokes. If ACST analysis was limited to ipsilateral stroke, the absolute benefit would be reduced [12].

CEA should be performed sooner rather than later in symptomatic patients. A pooled analysis of data from NASCET and ESCT demonstrated that the greatest absolute risk reduction for ipsilateral stroke, any stroke, and death within 30 days of surgery was found in patients who underwent surgery within 2 weeks of their last event. This decrease in benefit from surgery was significantly more rapid in women than in men. The benefit of early surgery is explained by the natural history of carotid plaque in symptomatic patients [13].

In a study conducted by Harrison and Marshall [14], $66 \%$ of patients undergoing CEA within 4 weeks of their most recent event had thrombus overlying the carotid stenosis compared with $21 \%$ of patients waiting for a longer period. Plaque morphology studies have shown that patients with recent symptoms are more likely to have acute plaque disruption, spontaneous embolization, overlying thrombus formation, and abnormal levels of metalloproteinases within the plaque. Ideally, patients presenting with a suspected TIA should have undergone investigation and, if appropriate, surgery within 2 weeks of presentation. Rothwell et al. [13] found that only 50\% of patients had undergone a duplex scan within 12 weeks of presentation, let alone surgery.

The best care of a patient with a progressing stroke of less than $24 \mathrm{~h}$ duration is less clear. Chaturvedi et al. [12] identified four studies that examined this issue. Three of the studies found benefit from surgery, but one reported a postoperative stroke and death rate of $20 \%$.

Practice varies widely between surgeons; some routinely insert shunts in all patients, whereas others eschew their use altogether. A middle way is a policy of selective shunting based on one or another monitor of cerebral function or blood flow. The technologies used to monitor for cerebral ischaemia are discussed below. There are limited high quality data to guide practice. The issue is made complex by the number of different options to be compared [15].

Studies may compare a policy of shunting vs not shunting, shunting vs selective shunting based on the use of a cerebral monitor, or selective shunting vs not shunting. [16] systematically reviewed the evidence for and against shunting. They found only three studies that were sufficiently rigorous for inclusion in a systematic review. Royal College of Physicians of London [17] compared routine shunting with no shunting; there was no significant difference in the rate of all strokes, ipsilateral stroke, or death up to 30 days after surgery. [18] compared shunting on the basis of EEG monitoring and carotid pressure measurement 
with pressure measurement alone. There was no significant difference between the risks of ipsilateral stroke between the two groups.

\subsection{Perioperative complications}

The two most feared major perioperative complications of CEA are cerebrovascular accident and myocardial infarction. The pooled data from the NASCET, ESCT, and Veteran's Administration Trial provide an insight into the incidence of cerebrovascular accident after CEA. Data are presented on 3248 patients who underwent surgery. Of 35 deaths [1.1\%] within 30 days of surgery, 20 deaths were from operative strokes. Two hundred and twenty-nine patients suffered the combined outcome stroke or death within 30 days of surgery [13].

The majority of perioperative events become manifest within $8 \mathrm{~h}$ of surgery. In a retrospective review of 771 CEAs, 26 patients developed a neurological deficit after surgery. Nineteen of the neurological deficits were diagnosed in the operating theatre or recovery room and a further five within $8 \mathrm{~h}$ of surgery [19].

\section{Anesthetic considerations for carotid endarterectomy}

Carotid endarterectomy [CEA] has shown a significant benefit in preventing ipsilateral stroke when it is compared to conservative management. Surgical morbidity and mortality must be kept to a minimum to achieve this benefit. Neurological status of the CEA patients can be monitored easily during regional anesthesia depending on the awake testing [neurocognitive assessment] method, and completely monitored, most medical centers consider awake testing as an essential method. [20].

\subsection{Anesthesia}

The awake patient's response test is a superior technique for monitoring patient's need for shunting during CEA. Because the awake assessment method used during regional anesthesia can avoid any shunts malfunction incidence [shuntrelated stroke] that may occur in GA with routinely shunt using protocol during CEA procedures. Besides, CEA procedures using GA increases postoperative complications in those patients with myocardial issues [21].

\subsubsection{General anesthesia}

The main advantages of GA are a safe airway throughout the procedure with controlled ventilation and arterial carbon dioxide concentrations. Anesthesia can be maintained using inhalational or i.v. agents. There are theoretical neuroprotective effects of GA agents [inhalational and i.v.] and some hemodynamic advantage of propofol anesthesia during carotid clamping. However, there is no outcome-based evidence favoring any particular general anesthetic agent during carotid clamping [22].

In a report by Stoughton et al. [23], 208 consecutive carotid endarterectomies were prospectively evaluated for cerebral function during surgery under regional anesthesia with simultaneous mental status evaluation and intraoperative electroencephalographic [EEG] monitoring. They found a high incidence of false positive [6.7\%] and false negative [4.5\%] EEG results in the detection of neurologic deficits when compared to an awake mental status evaluation. If the correlation is so bad in an awake patient, one must wonder what the utility is in an anesthetized patient. Assessment of neurologic function in the awake individual avoids all ambiguity in other surrogate testing modalities.

\subsubsection{Local anesthetic techniques}

Blockade of the second, third and possibly fourth cervical dermatomes is required for surgery. This can be achieved by various techniques; cervical epidural, superficial and deep cervical plexus blocks [alone or in combination] and local infiltration by the surgeon. There have been no differences in outcome demonstrated between these techniques [24].

\subsubsection{Cervical epidural}

Although this technique is not familiar to most anaesthetists, it has found favour in a few areas, in particular France. Commonly observed problems are hypotension, bradycardia and alterations in respiratory function. The reported dural puncture rate is $0.5 \%$ and respiratory failure requiring intubation occurs in around $1 \%$. These risks are unacceptable when safer techniques are available [24].

\subsubsection{Superficial cervical plexus block}

Conventional teaching is that local anaesthetic injected deep to the investing fascia of the neck cannot block the roots of the cervical plexus due to the impenetrable layer of deep cervical fascia. However, a recent cadaveric study demonstrated that an injection of methylene blue deep to the investing fascia did reach the nerve roots. This supports the observation that carotid surgery can be performed with the intermediate cervical plexus alone [24].

\subsubsection{Local anesthetic infiltration}

The operation can be performed under local anaesthetic infiltration by the surgeon [24].

\subsection{Monitoring techniques}

Almost all practitioners agree that there is a need to monitor $\mathrm{CBF}$ during $\mathrm{CEA}$, particularly during the period of arterial crossclamping. It is also agreed that the 'gold standard' in cerebral monitoring is to keep the patient awake during 
surgery so that sensory, motor, and higher mental functions can be assessed continuously. Any symptoms from reduced CBF or embolization are immediately apparent and this is one of the major benefits of performing surgery under local or regional anaesthesia [RA]. Several other monitors of cerebral perfusion are available including transcranial Doppler [which also detects the occurrence of microemboli], stump pressure measurement, EEG, somatosensory evoked potentials, and near-infrared spectroscopy. These electromechanical monitors are often used to determine the need for a temporary shunt, though their reliability in this regard varies [15].

\subsubsection{Vascular evaluations using stump pressure}

Stump pressure is a technique used to evaluate the need for shunting during CEA surgeries; this method is depending on measuring the back pressure in the distal part of the internal carotid artery after clamping. As the recorded reading of the back pressure in the targeted artery, the need for shunting was decided. Stump pressure values $>25 \mathrm{mmHg}$ shows the safe level as it was thought in the past years, later it was revised to mean of 40 $\mathrm{mmHg}$ as the safe level [25].

\subsubsection{Somatosensory evoked potentials}

Somatosensory evoked potentials [SSEPs] offer theoretical advantages over the EEG for cerebral ischaemia monitoring. This type of monitoring examines not only the cortex but the deeper structures of the brain. [26].

\subsubsection{Near-infrared spectroscopy}

Near infrared spectroscopy [NIRS] gives a value for regional cerebral oxygenation [rSO2] which is a composite measure of arterial venous and capillary oxygenation, although the predominant influence is the venous blood. Carotid cross-clamping produces a decrease in rSO2. Unfortunately, this change is not consistently related to changes in other measures of cerebral blood flow [27].

There is no well-defined 'normal' range for rSO2 during surgery and the change in rSO2 during a carbon dioxide challenge differs between two commercially available monitors [28]. Finally, there is not a well-defined biologic zero and studies in dead subjects give an average $\mathrm{rSO} 2$ value of $51 \%$, compared with a value of $68 \%$ in normal controlled subjects [29].

\subsubsection{Transcranial Doppler monitoring}

TCD ultrasonography was introduced in clinics in the early eighties for the first time. TCD is a noninvasive monitoring technique involving the use of $\leq 2 \mathrm{MHz}$ transducers, placed on the skull of the patient, through temporal bone windows [relatively thin bone] to measure the cerebral blood flow velocity and its alterations. [30].

\subsubsection{INVOS ${ }^{\mathrm{TM}}$ cerebral oximeter monitoring}

INVOSTM monitoring is an adjunctive noninvasive method which is intended for regional rSO2 monitoring in the brain or other targeted tissue through a sensor. The INVOS monitoring can be considered as an initial diagnostic method only but not used for confirming diagnostic purposes. [31].

\subsection{Issues beyond stroke, myocardial infarction, and death rates}

Although there were no significant differences between the two types of anesthesia in regard to stroke, MI and death, it may be appropriate to consider other additional parameters when comparing the outcomes of these two techniques. Thus, it analyzed the stress response that may be associated with adverse systemic effects and showed that patients under RA were exposed to higher stress levels, reflected as a rapid and significant increase in intraoperative values of cortisol and adrenocorticotropic hormone [ACTH]. However, in the early and late postoperative periods, the anesthetic modality no longer has an impact on surgical stress [32].

\section{General anesthesia or regional anesthesia}

A large number of studies compare RA and GA techniques in CEA surgery patients. The primary outcome was the proportion of patients with stroke [including retinal infarction], myocardial infarction, or death. One of the first major published studies included 803 consecutive CEAs [632 were performed under RA and 171 under GA] and showed that there was no statistically significant difference in the incidence of perioperative stroke or perioperative death between the two groups. However, the incidence of nonneurologic, nonfatal complications [eg, MI and postoperative mechanical ventilation; neck hematomas and cranial nerve injuries; and urinary retention] was significantly lower in the RA group [33].

In an attempt to solve the dilemma which anesthetic technique is superior, the randomized GALA trial recruited over 3500 patients with symptomatic or asymptomatic carotid stenosis from 95 centers between 1999 and 2007 to undergo CEA under GA or RA. Despite the enormity of the undertaking, the GALA trial results have not silenced the debate as to which anesthetic technique gives the best results. This study showed that there was no statistically significant difference in the incidence of stroke, MI, or death between randomization and 30 days after surgery. Actually, the trial was not powerful enough to detect a significant difference between GA and RA in major outcome [incidence of stroke and death]. The 
GALA trial showed significant differences between the 2 groups in terms of shunt insertion rate [14\% RA vs. $49 \%$ GA], but shunting was used purely at the discretion of the clinicians [at least in the GA group] rather than because of detected neurological deficit [34].

The choice of anaesthetic techniques remains guided by local clinical practice [surgeon and anaesthetist preference] as well as patient condition and motivation. With technical advances in ultrasonic imaging, evidence to support the widespread application of ultrasonic guidance for regional anaesthesia in orthopaedic and vascular interventions has been growing [35].

Several anaesthetic agents have also been incriminated in the reverse 'Robin Hood' syndrome in which abnormal cerebral vasodilation causes blood flow to be 'stolen' from active metabolic areas and diverted towards areas with lesser aerobic needs [36].

Disadvantages of regional anaesthesia are the need for patient collaboration, remote access to the upper airways and procedural complications such as temporary unilateral diaphragmatic and vocal cord paralysis, neural injuries and epidural, and subarachnoid or intravascular injection of local anaesthetic. Availability and experience with laryngeal mask airways offer new opportunities to maintain upper airways and satisfactory gas exchange under a moderate depth of anaesthesia while avoiding tracheal intubation [37].

Preoperative consultation plays a key role in identifying those patients unable to tolerate a 2hour procedure with the face and the neck covered by surgical drapes. Patients should be thoroughly informed regarding the anaesthetic and surgical approaches and emphasis should be placed on the importance of the 'awake' condition with verbal communication that contributes to the success of the intervention and offers the potential of preventing early neurocognitive dysfunction following CEA [38].

Maintenance of the 'awake' state offers the unique opportunity to detect almost instantaneously any intraoperative mismatch in regional brain oxygen supply. In addition, the release of stress hormones and arousal of the sympathetic system are helpful in preventing or mitigating brain ischaemia by keeping arterial blood pressure in the upper normal range, while avoiding the administration of vasopressive agents [39].

As viable cerebral tissues within the ischaemic penumbra are very sensitive to variations in blood pressure, improved early subclinical cognitive function after awake CEA [compared with general anaesthesia] could be attributed to better systemic haemodynamic control and preserved autoregulation of cerebral blood flow [40].

\section{References}

[1] HM. Goma, Ropivacaine, versus Ropivacaine Fentanyl for Carotid Endarterectomy. J Anesth Clin Res, Vol. 5, PP. 404,2014.

[2] A. Ciccozzi, C. Angeletti , C. Guetti, Regional anaesthesia techniques for carotid surgery: the state of art. J Ultrasound, Vol. 17, PP. 175$183,2014$.

[3] C. Verborgh , P. Van Den Brande, I. Van Heymbeeck, , UZ Brussel, Department of Anaesthesiology and Pain Medicine, Brussels, Cervical plexus block allows sooner hospital discharge after carotid endarterectomyBelgium euro journal of anesthesia,vol33,PP.360-365 1997.

[4] KittipanRerkasem, August 20, American Heart Association MD, PhD; Peter M. Rothwell, MD, PhD, FRCP, FMedSciLocal Versus General Anesthetic forCarotid Endarterectomy,Vol.66,PP.225-230,2009.

[5] A. Manbachi, On the shape of the common carotid artery with implications for blood velocity profiles. Physiol. Meas, Vol. 32,PP.367-370,2011.

[6] Ross LMMP, Atlas of anatomy. George Thieme Verlag,Vol.21,PP.225-230,2007.

[7] Vascular Disease Foundation. Carotid artery disease. Vascular Disease Foundation 8206 Leesburg Pike, Suite 301 Vienna, VA, Vol. 22,PP.182-185, 2012.

[8] RL. Sacco, R. Adams, G. Albers, Guidelines for prevention of stroke in patients with ischemic stroke or transient ischemic attack: a statement for healthcare professionals from the American Heart Association/American Stroke Association Council on Stroke: cosponsored by the Council on Cardiovascular Radiology and Intervention: the American Academy of Neurology affirms the value of this guideline. Stroke, Vol. 37, PP. 577-617, 2006.

[9] RW. Hobson, WC. Mackey, E. Ascher, Management of atherosclerotic carotid artery disease: Clinical practice guidelines of the Society for Vascular Surgery. J Vasc Surg, Vol. 48, PP. 480-6, 2008.

[10] American Heart Association, What is carotid endarterectomy? American Heart Association, Vol.19,PP.178-185,2015.

[11] N. Mohammed, SS. Anand, Prevention of disabling and fatal strokes by successful carotid endarterectomy in patients without recent neurological symptoms: randomized controlled trial. MRC asymptomatic carotid surgery trial ACST collaborative group. Lancet, Vol. 363, PP. 1491-502, 2004.

[12] S. Chaturvedi, A. Bruno, T. Feasby, Carotid endarterectomy- an evidence-based review: report of the Therapeutics and Technology Assessment Subcommittee of the American Academy of Neurology. Neurology, Vol. 65, PP. 794-801, 2005. 
[13] PM. Rothwell, M. Eliasziw, SA. Gutnikov, Endarterectomy for symptomatic carotid stenosis in relation to clinical subgroups and timing of surgery. Lancet, Vol. 363, PP. 91524, 2004.

[14] M. Harrison, J. Marshall, The finding of thrombus at carotid endarterectomy and its relationship to the timing of surgery. Br J Surg, Vol. 64, PP. 511-2, 1977.

[15] SJ. Howell, Carotid endarterectomy. British Journal of Anaesthesia, Vol. 99, PP. 119-31, 2007.

[16] R. Bond, K. Rerkasem, C. Counsell, Routine or selective carotid artery shunting for carotid endarterectomy and different methods of monitoring in selective shunting. Cochrane Database Syst Rev, Vol.190,PP.552-560,2002.

[17] Royal College of Physicians of London. Royal College of Physicians National Sentinal Stroke Audit vol36,PP.340-355,2004.

[18] JP. Fletcher, JG. Morris, JM. Little, LZ. Kershaw, EEG monitoring during carotid endarterectomy. Aust N Z J Surg, Vol. 58, PP. 285-8, 1988.

[19] MK. Sheehan, WH. Baker, FN. Littooy, MA. Mansour, Timing of postcarotid complications: a guide to safe discharge planning. J Vasc Surg, Vol. 34, PP. 13-6, 2001.

[20] Z. Gyöngyösi, L. Molnár, B. Fülesdi, Cerebral oxigen saturation and middle cerebral artery flow velocity changes during carotid endarterectomy. J Cardiothorac Vasc Anesth, Vol. 30, PP. 19-20, 2016.

[21] L. Pasin, P. Nardelli, G. Landoni, G. Cornero, S. Magrin, Y. Tshomba, Examination of regional anesthesia for carotid endarterectomy. J Vasc Surg, Vol. 62, PP. 631-40, 2015.

[22] D. Unic-Stojanovic, D. Babic, V. Neskovic, General Versus Regional Anesthesia for Carotid Endarterectomy. J Cardiothorac Vasc Anesth, Vol. 27, PP.1379-83, 2013.

[23] J. Stoughton, RL. Nath, WM. Abbott, Comparison of simultaneous electroencephalographic and mental status monitoring during carotid endarterectomy with regional anesthesia. J Vasc Surg, Vol.28, PP. 1014-23, 1998.

[24] L. Barclay, AAN Updates Guidelines on Carotid Endarterectomy Neurology, Vol. 65, PP. 794-801, 2005.

[25] S. Shahidi, A. Owen-Falkenberg, B. Gottschalksen, Clinical validation of $40-\mathrm{mmHg}$ carotid stump pressure for patients undergoing carotid endarterectomy under general anesthesia. J Cardiovasc Surg Torino, Vol. 58, PP. 431-8, 2017.

[26] TJ. McCulloch, CL. Thompson, A randomized crossover comparison of the effects of propofol and sevoflurane on cerebral hemodynamics during carotid endarterectomy. Anesthesiology, Vol. 106, PP. 56-64, 2007.
[27] Y. Ogawa, K. Iwasaki, S. Shibata, J. Kato, The effect of sevoflurane on dynamic cerebral blood flow autoregulation assessed by spectral and transfer function analysis. Anesth Analg, Vol. 102, PP. 552-9, 2006.

[28] K. Yoshitani, M. Kawaguchi, K. Tatsumi, K. Kitaguchi, H. Furuya, A comparison of the INVOS 4100 and the NIRO 300 near-infrared spectrophotometers. Anesth Analg, Vol. 94, PP. 586-90, 2002.

[29] G. Schwarz, G. Litscher, R. Kleinert, R. Jobstmann R, Cerebral oximetry in dead subjects. J Neurosurg Anesthesiol, Vol. 8, PP. 189-93, 1996.

[30] JJ. Chang, G. Tsivgoulis, Diagnostic accuracy of transcranial Doppler for brain death confirmation: Systematic review and metaanalysis. AJNR Am J Neuroradiol, Vol. 37, PP. 408-14, 2016.

[31] A. Denault, A. Deschamps, JM. Murkin, A proposed algorithm for the intraoperative use of cerebral near-infrared spectroscopy. Semin Cardiothorac Vasc Anesth, Vol. 11, PP. 27481, 2007.

[32] MM. Marrocco-Trischitta, A. Tiezzi, MG. Svampa, G. Bandiera, S. Camilli, F. Stillo, at al. Perioperative stress response to carotid endarterectomy: The impact of anesthetic modality. J Vasc Surg, Vol. 39, no. 6, PP. 1295-304, 2004.

[33] AK. Papavasiliou, HB. Magnadottir, T. Gonda, D. Franz, Clinical outcomes after carotid endarterectomy: Comparison of the use of regional and general anesthetics. J Neurosurg, Vol. 92, PP. 291-6, 2000.

[34] SC. Lewis, CP. Warlow, AR. Bodenham, B. Colam, PM. Rothwell, D. Torgerson, et al. General anaesthesia versus local anaesthesia for carotid surgery GALA: A multicentre, randomised controlled trial. Lancet, Vol. 372, PP. 2132-42, 2008.

[35] M. Lobo, Mourao J, AfonsoG. Carotid endarterectomy: review of 10 years of practice of general and locoregional anesthesia in a tertiary care hospital in Portugal. Braz J Anesthesiol, Vol. 65, PP. 249-254, 2015.

[36] AV. Alexandrov, VK. Sharma, AY. Lao, Reversed Robin Hood syndrome in acute ischemic stroke patients. Stroke, Vol. 38, PP. 3045-3048, 2007.

[37] JG. Augoustides, BE. Groff, DG Mann, Difficult airway management after carotid endarterectomy: utility and limitations of the laryngeal Mask Airway. J Clin Anesth, Vol. 19, PP. 218-221, 2007.

[38] CF. Weber, H. Friedl, M. Hueppe, Impact of general versus local anesthesia on early postoperative cognitive dysfunction following carotid endarterectomy: GALA Study Subgroup Analysis. World J Surg, Vol. 33, PP. 1526-1532, 2009. 
[39] J. Hoefer, E. Pierer, B. Rantner B, Ultrasoundguided regional anesthesia for carotid endarterectomy induces early hemodynamic and stress hormone changes. J Vasc Surg, Vol. 62, PP. 57-67, 2015.
[40] EJ. Heyer, JL. Mergeche, ZH Anastasian, Arterial blood pressure management during carotid endarterectomy and early cognitive dysfunction. Neurosurgery, Vol. 74, PP. 245$251,2014$. 